\title{
Criteria, Use Cases and Effects of Information Technology Process Automation (ITPA)
}

\section{Han Ping Fung*}

School of Management, Asia e University, Kuala Lumpur, Malaysia

\begin{abstract}
More and more IT organizations or IT outsourcing service providers are embracing Information Technology Process Automation (ITPA) as it has transformed the way IT services are delivered to end users. As a result, one of the highly claimed benefits of ITPA is total cost reduction in IT operations. However, ITPA subjects to different interpretations on topics like ITPA criteria, use cases as well as its effects on organizations. Today, there is limited literature and conflicting views on such ITPA topics. The objective of this study is to shed some lights on ITPA criteria, use cases as well as the effects ITPA brings. The effects include the benefits as well as negative effects of ITPA. Moreover, some factors to guide organizations how to adopt and deploy ITPA are also discussed. This study has taken a qualitative approach in which ITPA literature was reviewed and some IT professionals were interviewed. Lastly, limitations of the study, future research and conclusion were also provided.
\end{abstract}

Keywords: Information technology process automation; ITPA criteria; ITPA use cases; ITPA benefits; ITPA negative effects; Server automation; Storage automation; Network automation; Application automation; Security automation; Account identity and password automation; Automated job scheduling; ITPA integration

\section{Background}

Today, Information Technology Process Automation (ITPA) has improved the way IT organizations or IT outsourcing service providers in delivering their IT services to end users. Albeit IT by itself has transformed the way organizations are handling their businesses since the middle of 20th century, but the proliferation of ITPA has further reduced the total cost of ownership in managing IT [1].

The word "process" refers to a set of tasks designed to transform one or more inputs into outputs [2]. A process might include roles, responsibilities, tools and management controls to generate the outputs. A process might also define policies, standards, guidelines and work instructions as required.

The word "automation" is derived from the word "automatic" which means self-thinking or self-moving in Greek [3]. The word "automation" is popularized in manufacturing industry whereby production, movement and inspection are directed by self-operating machines without human intervention.

According to Nizri [4]. ITPA refers to IT capabilities that automate system and network operational processes while interacting with elements like applications, databases and hardware infrastructure. Technology adoption plays an important role in contributing to ITPA's success as technology within ITPA can help organizations to automate the remediation of alerts, incidents and increase their infrastructure uptime as well as reduce their operational costs [1].

It is worthy to note that ITPA is not the same as Information Technology Infrastructure Library (ITIL) or Information Technology Service Management (ITSM) Process Automation. In fact ITPA is a subset of ITIL or ITSM process automation. This is because ITPA's system and network operational processes are subsets of many processes within ITIL v3 Service Operation book [2]. Service Operation is only one of the five books of ITIL v3 which also include Service Strategy, Service Design, Service Transition as well as Continual Service Improvement books. Each of these books encompasses many more
ITIL or ITSM processes. Various technologies are used to facilitate ITIL or ITSM process automation [5,6].

Despite ITPA have different synonyms like Run Book Automation and Robotic Process Automation, but their definitions are similar to the ITPA. Johnson [7] has posited that run book automation refers to capabilities to support system and network operational processes through defining, building, orchestrating, managing as well as reporting the workflows pertaining to the processes. According to Institute for Robotic Process Automation, robotic process automation is the application of technology to enable employees to configure a "robot" or computer software so that it can interpret and control existing applications in processing transactions, manipulating data, triggering responses as well as communicating with other digital systems (www.irpanetwork.com). In a similar context according to Slaby [8], robotic process automation is the technological replacement of human worker to achieve efficiency through low cost software that runs as a web service, a scheduled task on a virtual machine / cloud services infrastructure, or a dynamically trigger-based process using existing workflows or messaging systems. In this study, the term ITPA from Nizri [4] is adopted instead of run book automation or robotic process automation due to its simpler operational definition, ease of understanding as well as the term ITPA encompasses wider spectrum of use cases.

ITPA is different from terms like IT-based Automation, Office Automation and Business Process Automation whereby ITPA is very much IT process oriented. According to Johnson [7], ITbased automation refers to the adoption of IT-based control system to reduce the need for human efforts which include muscular,

*Corresponding author: Fung HP, School of Management, Asia e University, Kuala Lumpur, Malaysia, Tel: 6012-6678119; E-mail: hanping.fung@aeu.edu.my

Received July 02, 2014; Accepted July 21, 2014; Published July 23, 2014

Citation: Fung HP (2014) Criteria, Use Cases and Effects of Information Technology Process Automation (ITPA). Adv Robot Autom 3: 124. doi: 10.4172/21689695.1000124

Copyright: (C) 2014 FUNG HP. This is an open-access article distributed under the terms of the Creative Commons Attribution License, which permits unrestricted use, distribution, and reproduction in any medium, provided the original author and source are credited. 
sensory and mental efforts in the production of goods and services. Office automation encompasses a wide variety of computer-based technologies that facilitate productivity of office workers which include: word processing, spreadsheet, presentation software as well as tools like email, instant messaging, voice communication systems and others $[9,10]$. According to Forrester Consulting [11], business process automation is the adoption of IT to automate back-office and frontoffice processes pertaining to accounting, financial, human resource, contact center and others.

The following example illustrated the differences among ITPA, ITbased automation, office automation and business process automation. Email service can be a form of IT-based automation in which email is replacing postman's slow mail service. At the same time, email can be a form of office automation service to facilitate the communication between office workers with other email users to improve their productivity. Moreover, email also can be embedded as part of contact center business process automation to notify management for a status update, escalation or request for approval. Previously, in order to maintain high availability of email service for end users, IT staff need to constantly involve in resetting passwords or unlocking email accounts when end users forgotten their password or their email accounts have been locked-up. Moreover, when end users' email accounts have exceeded the quota size assigned, IT staff needs to manually coordinate with end users to free up email server disk space. Today, these laborious works can be easily handled if ITPA is in place.

Problem statements triggered this study include: (a) it is unclear from literature what are the IT process criteria that justify for ITPA, (b) there are limited use cases of ITPA from literature as there is gaining interest in ITPA among IT organizations and IT outsourcing service providers, (c) there are conflicting views on the benefits of ITPA as some scholars disagreed on the benefits derived from ITPA. Hence, objective of this study is to provide some insights by clarifying the problems as mentioned above through a qualitative research.

Qualitative content analysis is adopted in this study whereby literature was searched and 37 IT professionals were interviewed in Klang Valley, Malaysia. The location Klang Valley was selected because it is one of the most developed locations in terms of ITPA projects deployment in Malaysia. The interviews were guided by preprepared semi-structured questions and were conducted after some IT organizations had implemented their ITPA projects. This article intends to address some questions raised pertaining to: (a) What are the IT process criteria that justify for ITPA? (b) What are the use cases for ITPA? (c) What are the benefits of ITPA? and (d) What are the negative effects of ITPA?

\section{Criteria For IT Process Automation}

This section of the study explores some of the IT processes that are suitable for ITPA. Characteristics of some IT processes might serve as the criteria for IT organizations or IT outsourcing service providers to look out for automation. From the literature reviewed and interviews with some IT professionals, there are nine workload characteristics for ITPA.

\section{High volume of transactions}

IT process workloads or their transactions which are voluminous are good criteria for ITPA [8]. Voluminous transactions are generally routine and repetitive whereby automation is an ideal choice. Moreover, voluminous transactions are easier to justify for automation due to its positive business case.

\section{High value of transactions}

IT process workloads with low volume but high value of transactions are also suitable for ITPA [12]. Normally low transaction volume is difficult to justify for investment from business case perspective. However, some management still approve the investment as the total cost of automation is still lower than the cost of business impact errors due to no automation. For example, in order to avoid paying hefty sum of penalty and meeting service level agreement of 24 hours a day and 7 days a week, some IT service providers still automate the IT processes to handle their limited customer transactions during weekends or holidays. This is because the cost of automation is still lower than the cost of penalty and human efforts during the weekends or holidays.

\section{Frequent access to multiple systems}

Processes that require IT staff to frequently access multiple systems in order to complete his or her jobs are also good candidate for automation [12]. Manual effort of such frequent access can lead to increased human errors, inconsistent performance and high cost of impacts. Hence, implementation of ITPA can help reduce such risk for IT organization or IT outsourcing service providers.

\section{Stable environment}

IT processes of a functional area in a stable environment can be another criteria for ITPA [8]. This is because IT processes in a non-stable environment can subject to uncertainties and expose to unpredictable disruptions. Hence according to [8], a period of 12 to 18 months without major changes to the IT processes is ideal for ITPA.

\section{Limited human intervention}

IT processes that required minimum human intervention can be a good candidate for automation [8]. This is because IT processes with grey areas that need IT staff to make adhoc decisions after detailed analysis and subjective judgment can render ITPA is not an ideal option for many organizations. However according to [12], IT processes with heavy human's subjective judgment and significant effort of human intervention should be also a candidate for ITPA. This is because the advancement of technologies like artificial intelligence can enable ITPA to model human-like thinking and subjectivity.

\section{Limited exception handling}

This criterion suggests that an ideal IT process destined for automation should handle exceptional cases minimally [8]. Despite robot or computer software of the IT process can be programmed to handle exceptional cases, the more exceptional cases the robot needs to handle can delay the process automation, testing and optimization. Hence, IT organizations or IT outsourcing service providers should opt for IT processes with fewer exceptional cases waiting to be handled. There is an opposite school of thought that proposed to automate IT processes with considerable number of exceptions handling [12]. This is because robot is best designed to handle exceptional cases due to the increased power of computing. Moreover, this school of thought also believes that the more the ITPA, the lesser the exceptional cases need to be handled.

\section{Manual IT processes prone to errors or re-works}

According to Sutherland [12], this criterion indicates that ITPA can add value by replacing manual IT processes which are error 
prone or unnecessary manual re-works are required. For example, batch job operations through manual scripting are susceptible to errors or mistakes created by human operator. Hence, ITPA should be considered by IT organizations or IT outsourcing service providers because not only ITPA can deliver at lower total cost of ownership but also at a more superior performance.

\section{Ease of decomposition into clear IT processes}

This criteria suggests that if an IT process which can be easily decomposed into clearly defined sub-processes, then automated modular business rules can be easily applied to achieve ITPA $[8,12]$. This is because clearly defined sub-processes with logical flows and unambiguity decisions to process inputs into outputs will facilitate ITPA.

\section{Clear understanding of current manual costs}

It is easier for IT organizations or IT outsourcing service providers to decide whether to implement an ITPA when the current cost of the manual IT process is known or documented. By conducting a business case, they can make a conscious decision to implement an ITPA when the future ITPA cost is lower than the current manual cost i.e. there is an expected cost saving [8]. Moreover according to Sutherland [12], building a business case by knowing the current manual cost might be easier to get support from management or stakeholders who might not well versed in the details of the robotic software or technology.

\section{Use Cases For IT Process Automation}

This section of the study illustrated some of the use cases for ITPA. The ITPA can be implemented using variety brands of robot or computer software which are easily available from the market. Rationale for these use cases is to enable one to evaluate how appropriate to implement or improve the ITPA in their organizations.

\section{Server automation}

There are some IT server processes that can be automated via ITPA. Several of them are depicted in the following sections.

Automate server provisioning and de-provisioning: This automated server process includes capability to perform automatic server discovery, server image (physical or virtual machine) snapshots capturing and cloning, server image, operating system and software provisioning / de-provisioning, operating system patching, audit and compliance evaluation, application configuration and deployment as well as reporting [13]. These automations can help IT organizations or IT outsourcing service providers to make changes more safely and consistently, thereby reducing unnecessary server down time. Server provisioning / de-provisioning automation is not only meant for production servers but also include development, test and disaster recovery servers whereby same servers can be deactivated, activated and re-used for next development project or disaster recovery exercise.

Automate server restart and shutdown: This automated server process includes the automation of servers and services restart, shutdown, reboot and reconfiguration of various types of servers. This ITPA can help organizations to reduce their energy consumption and IT operational costs significantly. According to Nizri [14], this server process is viable because workflows can be programmed to turn all network-connected idle servers into standby mode based on certain rules and policies. The switching of servers to standby mode is a preferred option than forced shutdown because standby mode minimizes the complications as well as reduces the need for long wait after cold server boot up.

There are occasions whereby servers need to be restarted and shutdown. These include frequent changes on application configuration files, server crashes e.g. email and backup servers or there is a need to release a server's memory. In order to address the abovementioned automatically, IT organizations or IT outsourcing service providers can setup workflows that are either triggered based on schedule or triggered automatically via alert. The workflows encompass monitoring, diagnosing, troubleshooting, sending an alert via email or Short Message Service (SMS) in case a server is down or a service is failed. In responding to these, the ITPA can either restart the server or service automatically, or wait till an email or SMS sent out by the IT staff. According to Nizri [14], automating a server or service restart can minimize risk as well as the application down time by $90 \%$.

Automate server disk space clean up: The monitoring and maintaining of servers with disk clean up can be a repetitive and time consuming activity if it is performed manually. However, this activity is crucial because running out of disk space due to growing log or temporary files in servers is one of the common causes for servers to crash and unscheduled downtime for many applications like Microsoft Structured Query Language (SQL), Internet Information Services (IIS) and Exchange [14].

In order to free up server disk space automatically, IT staff can automate workflows which can monitor servers for low disk space. When low disk space is detected, this automated process can alert the IT staff, archive, delete or move logs or temporary files to a different location. This automated process can be scheduled to run regularly or to be activated upon certain thresholds are reached.

Automate server backup and restore: Instead of performing manual server backup or restore which is repetitive and error prone due to human mistake, this automated server process can be programmed once into workflows and they can be executed automatically and accurately. The execution can be scheduled or activated upon certain events occurred.

Moreover, IT staff are recommended to capture a snapshot of the virtual server when they are performing risky changes or testing new software in the virtual server [14]. Snapshots can be lifesavers when patching or upgrading applications or servers. A virtual server snapshot is a copy of the virtual server at a certain point in time. The snapshot preserves the system memory and file system of the virtual server by enabling fallback to the snapshot in case something goes wrong on the virtual server.

Creating or deleting a snapshot can be automated by creating a policy and designing a snapshot workflow process. The workflow process can be triggered upon a backup policy is activated. The process includes reading a list of target virtual servers, creating snapshots for selected virtual servers, scanning server disk space availability, deleting snapshots if they are not needed in order to preserve disk space for new snapshots.

Automate file and folder handling: In day-to-day IT operations, IT staff can face voluminous of works to handle files and folders in various servers. By deploying robotic software, IT staff can automate mission critical application folders, files, events or log files monitoring, analysis as well as application or server self-correction [14]. Moreover, the robotic software can also perform automated secured file transfer, 
file availability check, comparing, archiving, adding, removing and renaming files and folders. For instance, in order to automate files and folders handling, pre-defined set of workflows can be enabled to monitor changes while performing file parsing and data integrity check. Changes can be detected based on attributes like file deletion, file size and date of last modification. The workflows can then handle any change or error with automated alert notification or remediation task as needed. Remediation includes deleting, moving, archiving, compressing files or others to maintain system availability.

\section{Storage automation}

Besides capability to perform automated storage provisioning and de-provisioning especially in a cloud computing environment like Storage-as-a-Service and storage thin provisioning, there are other automated storage processes as part of ITPA which include automated storage disk space clean up as well as automated storage backup and restore.

There is also an automated storage process which can increase or decrease storage space available to different servers from a pool of storage resources. This automated storage process can be executed automatically when some conditions are set and met.

Moreover, the movement of application data from highperformance to low-performance storage disks or vice-versa can be automated based on some application input-output metrics or statistics. The metrics or statistics can include perimeters like data age and frequency of access to those data.

\section{Network automation}

There are several use cases for automated network processes as part of ITPA. These include the pushing of new or updated configuration settings, performing operating system upgrades as well as deploying patches to network devices like switches, routers, gateways and others [15]. These network changes can be logged for tracking and auditing purposes. Moreover, another automated network process can be programmed to analyze and discover poor configurations throughout the network without human intervention so that remediation can be deployed before they affect the entire network performance.

\section{Application automation}

There are numerous use cases for application automation in ITPA. Some of them are illustrated in the following sections.

Database and middleware automation: This automated process incorporates robots or software that automate some administrative activities pertaining to database and middleware provisioning, configuration, compliance verification, patching as well as release administration [1]. If this day-to-day operation process is performed manually by IT staff or database administrators, it will take up much of their times, error prone and difficult to scale. On the contrary, automating this process will improve their productivity and delivery quality. The robot consists of workflows that perform specific automated tasks like provisioning, patching, health checking, defragmenting, compliance checking for all database or middleware servers. The IT staff or database administrators can also pre-specify the environment with specific information required by the workflows through configuring their perimeters.

With database and middleware automation, IT operators or even end users can execute routine yet complex database and middleware tasks. This will enable database and middleware subject matter experts can spend more times to define, enforce and audit the full stack of automation across different databases and middleware. For example, instead of manually and repetitively executing SQL queries to extract data and emailing them to designated users, automation can help database administrators or even empower end users to trigger this automated task [14]. This is achievable by creating a robot or automated workflow that connects and extracts data from the SQL database, generates and formats the data into required outputs e.g. text format or in spreadsheet file before sending them via email or SMS to the targeted end users. The automated workflow can also incorporate actions like email or SMS alert to database administrators in case any error is detected.

Email automation: Besides auto-emailing as per the example shown in previous section, there are other automations related to email. These include automate the adding of new user to shared mailbox or increasing email quota. In order to add new user to shared mailbox automatically, a workflow can be created which includes series of tasks to search and verify the user as well as the shared mailbox before the user is added. In order to increase email quota automatically, another workflow can be developed whereby a user can start requesting from a self-service portal. The workflow can include series of tasks like checking the user's current usage, quota entitlement and availability of email storage space before executing or rejecting the change. Alert of successful increment or rejection with indicated reason will be notified to the email user.

Application testing automation: With the proliferation of many applications to better support business agility and at the same time to reduce software delivery costs, application quality professionals today need to test more with less [16]. However, manual tests are time consuming and laborious exercises. Hence, application quality professionals or testers can resort to automated testing robot or software which can speed up the test execution. Automated testing robot not only can perform 24 hours and 7 days a week, moreover test cases can be converted from existing manual to automated tests cases easily. This conversion capability encourages the reuse of existing manual test cases that yield faster test creation and reduce test maintenance.

Furthermore, some automated testing robots come with preautomated test cases yet customizable for commonly used applications in which this can further speed up the testing [16]. For end to end integration testing that cut across numerous functional areas or departments, some automated testing robots enable users or testers to combine individual test cases into larger test sets. The testers can place those test cases into an end-to-end automated process flow and then execute the entire test set instantly or based on a pre-defined schedule.

Application deployment automation: The deployment process for applications like web servers, portal servers, Lightweight Directory Access Protocol (LDAP), business applications and others can be complicated as their deployments are in unique sequence whereby inconsistency might result when the deployments are performed manually. Hence, application deployment automation via robot or software can improve speed and consistency, reduce failure rates, increase ability to make changes across multitudes of application servers, improve confidence to roll-back changes and minimize disruptions to application users.

The different objectives between IT Infrastructure operation and the application teams have slow down the application deployment process and created some quality issues [16]. The adoption of robotic software in Development Operations (DevOps) enables end-to-end 
process automation to rapidly and reliably deploy new application code changes from source to target servers across different environments i.e. development, testing, staging as well as production.

\section{Security automation}

IT security is not only to keep organizations secure but also to do so more efficiently through security automation. According to Potter [17], automated security processes include designing, configuring, deploying, auditing and maintaining the security of IT systems as well as the security infrastructure as automated as possible. Some security automation can be simply modifying how IT staff is interacting with the security systems while other security automation required custom hardware and software to enable the automation. Yet other security automation required the IT organizations or IT outsourcing service providers to adopt standards like Information Security Automation Program (ISAP) and Security Content Automation Protocol (SCAP).

ISAP is a program to enable automation and standardization of technical security operations [17]. ISAP's objectives include enabling standard-based communication of data vulnerability, customizing and managing configuration baselines for various IT products, assessing information systems and reporting compliance status, using standard metrics to weight and aggregate potential vulnerability impact as well as remediating the identified vulnerabilities. SCAP is a suite of protocols designed to facilitate automation of the roles and issues pertaining to security operations [17]. SCAP is using specific standards to enable automated vulnerability management, measurement and policy compliance evaluation.

Generally, automated security process is capable to perform health check and remediation on IT systems. This is attainable by archiving, monitoring the system data, determining status of the devices, agents, applications as well as automatically send alerts for remediation or restart the systems as appropriate. This automated process is also capable to document all the steps in the workflow for future investigation or statistical reporting [14].

In addition, security compliance regulations required server administrator account passwords to change every 30 to 90 days. This effort is laborious and time consuming if the changes are performed manually. According to Nizri [14], automating the change of server administrator account passwords can reduce time from minutes to seconds with zero error and meet compliance regulations. In order to perform the change, automated workflow can extract server or service list from configuration management database or encrypted inventory text file. Then account login password for each server or service will be changed and followed by server or service restarts. Later, reporting and notification will be generated upon successful or failure of the password change.

\section{Account identity and password management}

There are some IT processes cut across server, storage, network, applications and security that can be automated. One of them is user account identity and password management. This automated process encompasses tasks like account creation, reset and deletion. When onboarding new users to single or multiple services, applications, access groups or others, creation of the user's profile, credential or account identity can be automated. Likewise, time consuming repetitive user account deletion also can be automated.

According to Nizri [14], unlocking user accounts, password resets and changing passwords manually accounted almost $40 \%$ of a service desk agent's workloads. Moreover, frequent password resets together with complex password policy lead to increasing number of account lockouts in which this has further increased the service desk agent's workloads. The workload for each case reported includes telephone or other means of authentication, execution of reset, confirmation and documentation of the case closure.

IT organizations or IT outsourcing service providers can deploy automated workflow or robotic software to handle the entire process of unlocking user accounts, resetting passwords or changing passwords. This process started when a user initiates the resolution through a selfservice portal. After appropriate authentication, automated workflow will unlock the account or reset the password and then send it back to the user. At the background, service desk agent can view and control user actions if required. Such process has reduced support calls, reduced end user's downtime and enable service desk agent to have more time to focus on more important works. According to Nizri [14], automated password reset alone can reduce the service desk queries by more than $30 \%$. Hence, automating account identity and password management is one the ITPA that IT organizations or IT outsourcing service providers should embark on if they have not started.

\section{Automated job scheduling}

According to Gilbert [18], job scheduling is classified into three generations i.e. batch processing, workload management and workload automation. The first generation's batch processing refers to the scheduling of non-interactive jobs in order to optimize the usage of costly computing resources. Batch processing features include: automatic restart and recovery, file management, integration with security systems, operator alerts, scheduler failover, service classification, spooling of devices, scheduler throughput management as well as workload failover.

Later workload management which equipped with richer scripting features and more sophisticated scheduling engines can manage jobs distributed across numerous and diverse platforms from a central control point [18]. Workload management features encompass: crossplatform support, cyclical scheduling, deadline scheduling, interdependent jobs execution, dynamic resource balancing, external task monitoring, multiple calendars and time zones scheduling, on-demand scheduling, scheduling of packaged applications as well as scheduling of web applications.

The third generation's workload automation refers to more advanced job scheduler that is integrated with web-based applications and the scheduling of jobs based on real-time events. Through workload automation, Service Level Agreements (SLAs) can be defined for critical business services and their performance can be monitored against those SLAs [18]. Workload automation features include: scheduling based on conditional dependencies, critical-process monitoring, dynamic scheduling, event-based automation, graphical workflow definition, mobile access management, creation and execution of programmable scheduler Application Programming Interfaces (APIs), virtualization enablement, workload forecasting and workload planning.

\section{ITPA integration}

There is significant progress in respective server, storage, network, application and security process automation. However these silos' progress is less than ideal due to their silo-based automation. There is little cooperation between these silos and lack of consistency in these processes [4]. Hence, it is difficult to setup end to end services through ITPA. 
In order to manage IT operations with consistency, compliance as well as ensure timely delivery of IT infrastructure and application services, it is paramount important to integrate all these silos of automated processes. There is some orchestrator software in the market today which offer innovative ITPA platform that provide for integrated automation deployment and management with improved user experience [4]. This orchestrator software promotes different automation integration by coordinating different automated processes across teams, tools and environments. Some of these orchestrator software not only can integrate server, storage, network, application and security process automation, they are also capable to integrate with service desk ticketing system, monitoring system as well as some ITIL processes which are beyond the scope of this article.

\section{Benefits of IT Process Automation}

There are numerous benefits contributed by ITPA. These include: IT service predictability, IT service re-usability, better IT services integration, increased IT service productivity, reduced IT service risk, IT service cost effectiveness and improved business performance. Following sections explained how these benefits are achieved through ITPA.

\section{IT service repeatability}

IT service outcomes can be very different if they are delivered by different IT staff based on manual processes in which some procedures might be undocumented, unused or outdated. Worse still when those IT staff leave the IT organization in which they might take the core knowledge with them, leaving the IT organization struggling to deliver repeatable IT services.

However with ITPA, IT processes and procedures are represented as automated workflows [1]. These workflows just need to be created or captured once and they can be executed repetitively with the same results. Different or newly joined IT staff can deliver the same IT service outcomes by merely activating these automated workflows. Hence, IT organizations or IT outsourcing service providers can ensure IT services are delivered repetitively with the same results through ITPA. Moreover, the same automated workflows can be quickly and easily reused for different business units or in different IT organizations $[8,12]$.

\section{IT service predictability}

According to Hewlett-Packard [19], IT organizations or IT outsourcing service providers can attain a new level of predictability in their IT services through ITPA. This is because ITPA can standardize the IT processes and eliminate human error that occurs in manual processes. As a result, IT services become more consistent and accurate in which these will provide more predictable IT services to end users.

For example, IT administrators can initiate an automated workflow to check applications or servers for compliance status before trigger server, storage or network automation products to perform the required updates. The workflow can run across hundreds of servers or devices with consistency, accuracy, audit trail completion as well as documentation creation that the IT administrators required for compliance purposes [1]

\section{Better IT services integration}

Before the adoption and deployment of ITPA, IT system management products are not delivered at their full potential. Not only the products are partially deployed, most of them are not well integrated with other IT system management products. Moreover, most organizations are running heterogeneous products from multiple vendors which can be complex and expensive. As a result, IT systems management products are only achieving a mixed success [1].

However with leading ITPA products especially those come with integrated orchestrator, they can help IT organizations or IT outsourcing service providers to automate IT processes and integrate disparate products that support these processes [1]. Moreover, some of these ITPA products are capable to link-up various ITIL processes like incident, problem, configuration, event, capacity and availability management. Through automation and integration, this will lead to efficient, effective and proactive IT service delivery.

\section{Increased IT service productivity}

After implementation of ITPA, productivity of IT staff increased when delivering their IT services. ITPA enforces the adoption of IT standards by adhering to pre-defined procedures, communications and escalations in which these will reduce the alert floods $[1,4]$.

Moreover, ITPA also can minimize resolution time by $50-90 \%$ with faster response to critical IT events especially during off-duty hours. This enables IT staff to resolve more incidents per hour or higher productivity whereby manual tasks used to be completed in days can be achieved in hours. Such situations free up IT staff by allowing them to focus more on strategic IT initiatives instead of spending time on repetitive time-consuming tasks [4].

In addition, ITPA through robotic software can perform full time works in true 24 hours a day and 7 days a week [12]. Robotic software does not require to be paid over time and can be fully utilized to operate around the clock. The tireless robotic software work faster, more efficiently and with fewer errors. They do not need coffee breaks, holidays or even health insurance in comparison to IT staff. According to Slaby [8], a software-driven robot typically can replace the works of 1.7 IT staff.

\section{Increased IT services satisfaction}

According to Nizri [4], ITPA improves service quality up to $70 \%$ whereby IT staff can take actions automatically by responding to user requests. This is because ITPA can remove many mundane and unsatisfying tasks that are empowered to IT staff. With ITPA, this can serve as a means to retain IT staff and enhance their job satisfaction [12]. Enhanced job satisfaction can reduce the impact of attrition due to job dissatisfaction among IT staff. Moreover, ITPA also can promote customer satisfaction. According to HP, end customers of IT organization or IT outsourcing service provider might not necessary understand the sophistication of ITPA that have been implemented. However, they understand they are getting better services from IT so that they can get their jobs done faster and more effectively.

\section{Reduced IT service risk}

According to Forrester Consulting (2011), ITPA has reduced the IT service risk by lowering unauthorized data access and non-compliance as well as improving security and governance. Nizri [4], Slaby [8], HP [19] and Sutherland [12] has posited that ITPA can reduce IT service risk by facilitating the achievement of higher compliance to regulatory requirements through granular process monitoring, errors reduction, automatic reporting and auditable events trace-back. 


\section{IT service cost effectiveness}

IT organizations or IT outsourcing service providers can unlock the ITPA investments by reducing the cost to deliver IT services [11]. According to Sutherland [12], today ITPA can reduce the cost of an IT staff's Full Time Equivalent (FTE) by two thirds while the IT staff is completing a task or process manually. Hence, robotic FTE that can work around the clock without error which is one third the price of an outsourced FTE might encourage some IT organizations to in-source back their IT operations.

Moreover, lower cost of IT services is achievable with ITPA compares to totally new application or system transformations in which the latter will incur the higher IT cost of ownership. According to HP [19], ITPA can increase the Return of Investment (ROI) from existing assets of an IT organization. For example through ITPA, IT organizations can take advantage of technologies or techniques like "thin provisioning" of storage and server systems, allowing IT staff to reclaim actually unused capacity on those systems. This situation can improve the ROI of the existing resources and might enable IT organizations to avoid or delay new capital expenditure investment that would otherwise have been required.

\section{Improved business performance}

IT organizations or IT outsourcing service providers can leverage ITPA to deliver business values for their business organizations [12]. Through ITPA, the business organizations can transform their business outcomes by better support of contracting, reducing sales cycles, increasing working capital, improving customer satisfaction and others. Moreover, ITPA also enable business organizations to differentiate themselves from competitors through innovative new business solutions.

According to Adapt One [20], ITPA can short-circuit many of the manual tasks that slow down the overall efficiency of a business. In order to maintain competitive edge in the business world today, agility is at the heart of every business organization. For example, shipment deadlines need to be met and customers must be serviced within certain time frames. Hence, ITPA can contribute to a business organization's agility and eliminate many of the manual steps which are cumbersome to the overall business process.

\section{Negative Effects of IT Process Automation}

Despite some benefits depicted in the previous sections, at the same time ITPA also yielded some negative effects. Some of the negative effects include: job lost for some IT staff, IT staff need to be re-skilled, IT staff job complacency, lack of IT staff personal touch as well as higher cost of IT operations.

\section{Job lost for some IT staff}

According to Brown [21], automated technology inclusive ITPA has resulted in today's high IT staff unemployment and low hiring rates. For example, ITPA in the fields of call centers and airline ticketing services to medical diagnostic and corporate supply chains has replacing IT staff faster than the economy can create jobs. Instead of hiring IT staff, many IT organizations are investing in ITPA technology that boost productivity and expand output that previously were not attainable.

Moreover, previously IT staff was required as they could recognize complex patterns whereby computers were not up to expectations. IT staff understood what information to draw upon to help end users with complains or diagnose symptoms of an IT issue. Equally important, they also could communicate their findings with others. However, through automated technology like ITPA, Google's autonomous car, International Business Machines (IBM) Jeopardy-playing Watson supercomputer as well as software that are capable to analyze medical images for cancer, automated technology has replaced humans as the better pattern recognizer. Hence, IT organizations might not hire IT staff to do the job anymore whereby computers are getting better than humans [21].

\section{IT staff need to be re-skilled}

Based on the results of qualitative interviews with some IT organizations that have implemented ITPA in Klang Valley, one should not underestimate the additional responsibilities to maintain the ITPA tool after its implementation. This is because the existing IT staff might not have the skills or time to learn and maintain the automated processes. Hence, reskilling IT staff through lab training and on-the-job training is critical to ensure the successful deployment of an ITPA project. According to some large IT organizations, the role to design and automate new IT processes as well as managing ITPA can be a fulltime job.

\section{IT staff job complacency}

According to Singh et al. [22], the extensive use of automated systems inclusive ITPA has created a new concern whereby IT staff is not capable to detect an automation malfunction or being termed as "automation-induced complacency". The study conducted by Sing et al. [22] shown that high static automation reliability has increased automation-induced complacency and perceived mental workload has reduced from pre to post-test sessions under high static reliability condition. In order to address automation-induced complacency, appropriate strategy related to automated process design and training pertaining to human-automation interaction should be approached [23]. Another strategy to minimize automation-induced complacency is to adopt adaptive automation [22]. Adaptive automation is the modification of functionality, level or number of automated systems in real time to enable the resetting of the task environment based on evolving situational demands. Through adaptive automation, this will strike a balance between task demands and cognitive resources of the IT staff so that they are vigilant toward any automation malfunction. These two strategies to address automation-induced complacency are also important during a disaster recovery situation whereby automated IT processes might not be available and manual IT processes must be followed by IT staff.

\section{Diminishing of personal touch from IT staff}

Personal touch in a business context refers to the ability to deliver enhanced customer services as well as having better relationships with vendors and organizational systems which are conducive for human interaction [20]. According to some IT organizations that have implemented ITPA in Klang Valley, ITPA has reduced the personal touch of IT staff towards their end users. This observation is visible especially in large call centers or service desks whereby some of the IT works have been automated e.g. passwords reset automation. However according to Adapt One [20], when tasks are accomplished automatically, IT staff have more time and energy devoted toward customer satisfaction. Moreover, with fewer mistakes made this will enhance the relationships with customers. When this finding of Adapt One was shared with interviewees in Klang Valley, most of them have 
commented that once ITPA is deployed, the IT staff usually being redeployed to another role or being laid off.

\section{Costly deployment of ITPA}

According to some IT organizations that have implemented ITPA recently in Klang Valley, they discovered that the cost to implement ITPA was exorbitant high. The actual cost of implementation was higher than the planned cost due to requirement gaps and project overrun which should be contained at the earliest possible. They also commented that if the actual cost was tabulated for IT management approval before the project was initiated, approval to proceed would not be granted. Moreover, some IT organizations also commented that ITPA incurred higher IT operation costs due to higher skilled labor rates. However according to Forrester Consulting [11], HP [19] and Sutherland [12], total cost of ownership for long run which includes initial implementation cost as well as ongoing operation cost for ITPA is lower than the total cost of ownership before ITPA was implemented. Moreover, as IT organizations or IT outsourcing service providers automate more of their IT processes, the ROI will continue to grow.

According to a qualitative interview with two IT professionals from an IT outsourcing service provider, they commented that the IT operation costs based on manual labor are lower than the IT operation costs after deploying ITPA. The rationale is that the IT labor costs for some countries in Asia Pacific including Malaysia are relatively cost effective. Moreover, all the manual IT labors are classified into different skill levels and specializations e.g. basic level eyeballing of server availability whereby high turnover will be easily replaced by low cost IT staff. Hence, the deployment of ITPA is only logical when those countries' IT labor rates are high.

\section{Factors to Consider When Adopting and Deploying IT Process Automation}

\section{Justify with a business case}

In order to justify ITPA is the right approach to improve an organization's IT services, one should start with a business case. A business case essentially helps IT management or decision makers to have a fine-grained understanding of the current manual IT process costs versus the future ITPA's quantitative values. A business case is easy to setup when an IT organization have clear understanding of their current manual costs which is one of the criteria for ITPA as described in the previous section. A healthy business case will guide the IT organization to make a conscious decision to proceed with an ITPA project as there is a ROI. Without a business case, it is difficult to convince stakeholders to commit or support the ITPA project as one cannot quantify whether robotic software is cost effective than a human labor.

Before embarking on a business case, one should define the Key Performance Indicators (KPIs) for success. These include number of IT processes automated, number of hours saved, metrics of service response improvement and others. These KPIs serve as base-lined goals for IT management to compare the required future and current costs with or without ITPA in a later business case study.

In order to make a business case justification more realistic, one should also conduct a Proof of Concept (POC) or simulate a smaller scale of ITPA deployment so that all the costs are captured which include: hardware, software, one-off implementation, training, ongoing operation as well as maintenance costs. Through the use of business case and the complementary support from KPIs and POC, these might minimize the costly deployment of ITPA faced my some of the IT organizations as depicted in the previous section. This is because base-lined goals are set in a real ITPA environment whereby all the future and current cost components are considered and compared.

\section{Start small think big}

IT organizations are advised to start with simple IT process automation first. Rationale for this is that it is less complex, lower risk, lower cost, experience gaining and serves as a "quick win" with immediate result. According to Bennett [24], adopting ITPA by starting with simple IT processes not only enable IT staff learn how ITPA works, it can also help IT staff to evaluate how ITPA needs to be adapted according to an IT organization's requirements. One of the lessons learnt in this study is that an organization needs to carefully consider each ITPA criteria in its organizational context because unexpected criteria still justify the adoption of ITPA. For example non-voluminous transactions, frequent human intervention as well as frequent exception handling still qualify some organizations to adopt and deploy ITPA.

Once the ITPA learning curve increases, IT organizations can think big how to expand the adoption and deployment of ITPA into other departments, business units, subsidiaries or even other organizations. Even though an IT organization starts with small-scale ITPA projects, it can also expand in a modular fashion and apply ITPA into processes that cross domains like server, storage, network, security and others. Doing this can transforms the IT organization or even the business organization to operate from reactive to proactive mode.

\section{Use the right human resources}

In order to adopt and deploy ITPA successfully in an IT organization, tapping on the right human resources are paramount important. Before the funding of an ITPA is approved, the IT organization needs to secure top management buy-in whereby business case, KPIs and POC need to be completed. This is because business case showcasing the cost of robotic FTEs versus in-house or outsourced FTEs should get immediate buy-in from top management. However, IT organization also needs to address issues arise from certain stakeholders that might sabotage the ITPA project due to loss of control, loss of job and resistance to change [24].

Before an ITPA project starts, the IT organization needs to assess the skillset and deployment effort required from its IT staff. These include competency to setup, configure and maintain the automated workflows. If internal competency is inadequate, the IT organization should utilize experienced consultants especially on the first few IT processes. The objective is to save time, money and to obtain the best results from external consultants. Moreover, working with external consultants also enable internal IT staff to gain the required knowledge, methodology, trainings and experience so that they are competent to implement the future ITPA projects themselves [24].

During ITPA project implementation, IT organization needs to involve all the key personnel from the current IT process. Incorrect input or without their input, the ITPA project is doomed for failure as inefficient process flows are built and the same inefficiencies continue troubling the IT organization. Hence, before ITPA project starts, existing process key personnel should be engaged to conduct a thorough system or process analysis [4]. This includes documenting existing systems, processes, interfaces, integration points, input and output formats. The objective of this exercise is to enable the IT 
organization understands their current IT process before ITPA project is implemented.

\section{Conclusion}

\section{Answering study questions}

This study is guided by several questions posed at the beginning of the study. In answering what are the IT process criteria that justify for ITPA, this study has indicated nine criteria which include: (a) high volume of transactions, (b) high value of transactions, (c) frequent access to multiple systems, (d) stable environment, (e) limited human intervention, (f) limited exception handling, (g) manual IT processes prone to errors or re-work, (h) ease of decomposition into clear IT processes, and (i) clear understanding of current manual costs. As for what are the use cases for ITPA, this study has provided eight use cases i.e. server, storage, network, application, security automations, account identity and password management, automated job scheduling, and ITPA integration. This study also addressed another question pertaining to what are the benefits of ITPA in which these include: (a) IT service repeatability, (b) IT service predictability, (c) better IT services integration, (d) increased IT service productivity, (e) increase IT services satisfaction, (f) reduced IT service risk, (g) IT service cost effectiveness, and (h) improved business performance. On the other hand, in answering what are some of the negative effects of ITPA, this study has discovered five negative effects. These include: (a) job lost for some IT staff, (b) IT staff need to be re-skilled, (c) IT staff job complacency, (d) diminishing of personal touch from IT staff, and (e) costly deployment of ITPA.

\section{Limitations of the study}

There are some limitations in this study. Firstly, this study provided a high level view on ITPA use cases which did not drill down into detailed technicality. This study also tried to be ITPA product neutral and minimized the citation of ITPA vendor brands as well as their specific technologies. Secondly, albeit ITPA is a subset of ITIL or ITSM process automation [2], there is no detailed depiction on their differences and how they can be integrated. Thirdly, even though both IT organizations and IT outsourcing service providers are embracing ITPA, it is unclear whether IT organizations will still consider the outsourcing option in view that IT outsourcing service providers are also deploying ITPA as part of their solution to customers. Fourthly, this is a qualitative study in which some interviewed transcripts had been collected. However, due to the article length's constraint, the narration to capture how those comments emerging as main themes were not demonstrated. Last but not least, some of the interviewed comments from IT professionals in Klang Valley, Malaysia might not generalize to all IT organizations. These include comments on reskilling of IT staff, diminishing of personal touch as well as costly ITPA deployment.

\section{Future research}

One of the future researches that can be embarked on is the end to end studies of ITPA. This includes how ITPA started to influence IT services all the way into business or organizational-wide services. Such studies potentially can improve business performance of an organization and its funding approval should be easier to justify. Moreover, in depth studies of use cases combining ITPA and Business Process Automation (BPA) across various industries should be considered also. This is because they serve as useful process models to encourage the adoption and integration of ITPA and BPA. Secondly, detailed studies on the relationships, differences and integrations between ITPA and ITIL process automation can be conducted. Thirdly, further studies on IT organizations' motivation to outsource or embrace ITPA directly is worth researching as IT outsourcing service providers are also ramping up to embrace ITPA. Lastly, this study is based on qualitative interviews with 37 IT professionals. Future work should include empirical quantitative studies to determine how significant some of the criteria and use cases are influencing the ITPA adoption. Moreover, quantitative studies should be conducted to confirm how ITPA adoption has significant bearing on the benefits and negative effects of ITPA.

\section{Conclusion}

ITPA is gaining traction recently among IT organizations as well as IT outsourcing service providers due to its promising cost saving capability and enriched values it has contributed. Moreover, ITPA is a critical or core component in any cloud solution because ITPA enables cloud characteristics which include: broad network access, rapid elasticity, measured services, on-demand self-service as well as resource pooling [25]. However, every organization that wishes to adopt and deploy ITPA or cloud solution successfully needs to equip with the right knowledge to make the right decision throughout its ITPA journey. Hence, understanding of the ITPA criteria, use cases, benefits, negative effects as well as some factors to consider as illustrated in this article might empower the organization to do so.

\section{References}

1. Hewlett-Packard (2012) Top Ten Reasons to Automate Your IT Processes. Business White Paper, Hewlett-Packard Development Company.

2. Information Technology Infrastructure Library (2007) Service Operation. The Stationery Office.

3. Schey JA (2000) Introduction to Manufacturing Processes. McGraw-Hill Higher Education, USA

4. Nizri G (2012a) IT Processes Automation - Your Survival Guide!.Ayehu Software Technologies.

5. Ayachitula N, Buco M, Diao Y, Maheswaran S, Pavuluri R, et al. (2007) IT Service Management Automation - A Hybrid Methodology to Integrate and Orchestrate Collaborative Human Centric and Automation Centric Workflows. IEEE International Conference on Services Computing (SCC 2007)

6. O'Donnell G (2007) Pragmatic ITIL Process Automation - Why IT Operationa Excellence Requires Unifying Process and Technology. EMC Corporation, USA.

7. Johnson M (2011) Run Book Automation - What You Need to Know for IT Operations Management. Tebbo Publisher.

8. Slaby JR (2012) Robotic Automation Emerges as a Threat to Traditional Low Cost Outsourcing. HfS Research Ltd.

9. Olson $\mathrm{MH}$ Lucas $\mathrm{HC}$ (1982) The Impact of Office Automation on the Organization: Some Implications for Research and Practice. Communications of the Association for Computing Machinery 25: 838-847.

10. Parker C, Case T (1993) Management Information Systems: Strategy and Action Mitchell McGRAW-HILL, Watsonville, Canada.

11. Forrester Consulting (2011) The Role of IT in Business-Driven Process Automation. Forrester Research Inc, USA.

12. Sutherland TC (2013) Framing a Constitution for Robotistan - Racing with the Machine for Robotic Automation. HfS Research, Ltd.

13. Turner MJ (2012) IDC MarketScape: Worldwide Distributed Server / Workload Automation Software 2012 Vendor Analysis.

14. Nizri G (2012b) 10 Time-Consuming IT Tasks You Should Automate Today! Ayehu Software Technologies. 
Citation: Fung HP (2014) Criteria, Use Cases and Effects of Information Technology Process Automation (ITPA). Adv Robot Autom 3: 124. doi: 10.4172/2168-9695.1000124

Page 10 of 10

15. Illsley R, Rodger A (2011) CA Automation Suite for Data Centers. CA Technologies, Ovum Publisher.

16. Hewlett-Packard (2012b) Demands for Agility - Accelerated Test Automation. White Paper, Hewlett-Packard Development Company.

17. Potter B (2007) Security Automation - Better Living through Common Protocols Ponte Technologies LLC, USA.

18. Gilbert M (2006) Job Scheduling on Windows. White Paper, Legacy Directions.

19. Hewlett-Packard (2012c) Enlightened Data Center Automation in the Cloud Age - How to Achieve Transformative Efficiency Gains Without Disrupting IT. Business White Paper, Hewlett-Packard Development Company.

20. Adapt One (2010) Automate Processes for a Competitive Edge. White Paper Adapt One Company, London.

\section{Brown AS (2012) Automation vs Jobs. Mechanical Engineering.}

22. Singh AL, Tiwari T, Singh IL (2009) Effects of Automation Reliability and Training on Automation-Induced Complacency and Perceived Mental Workload. Journal of the Indian Academy of Applied Psychology 3: 9-22.

23. Lee JD, See KA (2004) Trust in Automation: Designing for Appropriate Reliance. Human Factors 46: 50-80.

24. Bennett A (2009) Best Practices for Process Automation - Achieve Early Success with the Processes Your Business Automates. White Paper Interactive Intelligence, Inc.

25. National Institute of Standards and Technology (2011). The NIST Definition of Cloud Computing. Special Publication 800-145. 